\title{
Perceived challenges in diabetic care: A cross sectional study among in-patients of a tertiary care hospital
}

\author{
Manjunatha S $\mathbf{N}^{1}$, Sajja Jayashree ${ }^{2^{*}}$ \\ ${ }^{1}$ Associate professor, ${ }^{2}$ Senior resident, ${ }^{1-2}$ Dept. of community Medicine, ${ }^{1}$ Mysore Medical College And Research Institute, Mysore, \\ Karnataka, ${ }^{2}$ BMCRI- Bangalore Medical College and Research Institute, Bengaluru, Karnataka, India
}

*Corresponding Author: Sajja Jayashree

Email: jaysaj2056@gmail.com

\begin{abstract}
Introduction: India recognized as capital for diabetes in world accounts for 69.2 million cases i.e. $8.7 \%$ as per the 2015 International Diabetes Federation Data. Despite the availability of efficient medications the mortality and morbidity due to the disease is increasing. Therefore the current study was carried with an aim to identify the barriers of access to care.

Objective: To identify the socio-cultural, economic and educational barriers in continuum of treatment among diabetics. Materials and Methods: A cross sectional study was conducted in a tertiary care hospital, Mysore. Diabetic patients admitted in the hospital were considered for the study.

Results: There was no fear of stigma among the participants. $80 \%$ of the patients were dependent either economically or physically on their family members, amongst which only half of them got the required family support. $83 \%$ of the participants felt that travel to hospital, stay and other expenses were adding on to their existing financial burden of direct and indirect economic loses. The proportion of people among literates who had good knowledge was higher as compared to illiterate but the difference was not statistically significant.

Conclusion: Counselling sessions must be provided both in hospital and community based setting to enhance health literacy among diabetics.
\end{abstract}

Keywords: Diabetes mellitus, Socio-cultural, Health literacy, Economic burden, Counselling.

\section{Introduction}

India is a land of diversity. India is a country with a diverse range of cultures, ethnicities, religions and languages. What are the perceptions about health among people hailing from different socio cultural, economic and educational backgrounds? What is their level of knowledge, what are their expectations and how far can they put in extra effort for the betterment of their health? Therefore managing an ailment requires understanding of the influence of these diversities which will in turn have more impact in chronic illness like diabetes. ${ }^{1}$

Globally, the prevalence of diabetes has nearly doubled since 1980 , rising from $4.7 \%$ to $8.5 \%$. An estimated 422 million adults worldwide of which 60 million from India, were living with diabetes in 2014. It is now recognized that, it is the low- and middle-income countries that presently face the greatest burden of diabetes. ${ }^{2}$ In 2019 approximately 463 million adults were living with diabetes; by 2045 this will rise to 700 million. The proportion of people with type 2 diabetes is increasing in most countries. $79 \%$ of these adults with diabetes were living in low- and middle-income countries. The greatest number of people with diabetes were between 40 and 59 years of age. 1 in 2 people with diabetes were undiagnosed. Diabetes caused 4.2 million deaths. More than 1.1 million children and adolescents are living with type 1 diabetes. More than 20 million live births ( 1 in 6 births) are affected by diabetes during pregnancy. 374 million people are at increased risk of developing type 2 diabetes. ${ }^{3}$

While in many ways the diversity is a source of richness and strength, cultural influences sometimes give rise to challenges in the context of managing commonly presenting illnesses. ${ }^{4}$ Awareness of diabetes and diabetic care is needed for successful disease management. The low level of awareness of diabetes and its complications among patients and physicians results in poor glycemic control. A patient-centric approach of diabetes care must be built, which is sensitive to the needs of a patient and avoids placing an undue burden of therapy on the patient. An accelerated process of negotiation and motivation should be initiated, support enlisted from family, friends and paramedical staff. It is known that improved well-being, self-care, and diabetes control are seen in patients who report more involvement in making treatment decisions. ${ }^{5}$

Since diabetes is an illness which is effecting whole community therefore it is a major public problem. Despite the availability of efficient medications the mortality and morbidity due to the disease is increasing. Therefore the current study was carried with an aim to identify the barriers of access to care.

\section{Objective}

To identify the socio-cultural, economic and educational barriers in continuum of treatment among diabetics.

\section{Materials and Methods}

A cross sectional study was conducted in a tertiary care hospital, Mysore. Diabetic patients admitted in the hospital were considered for the study. Convenience sampling method was adopted. Considering $8.7 \%$ prevalence (IDF 2017), at $95 \%$ confidence interval with an absolute error of $10 \%$ the calculated sample size was 97.100 participants were interviewed using a pretested semi structured questionnaire. Percentages and proportions were calculated 
to compute the results. Admitted patients who were severely ill requiring intensive care were excluded from the study. Diabetic patients who were diagnosed and were on medication for at least 2 years were included in the study.

\section{Results}

Mean age of the participants was 57 years. $69 \%$ of the participants were females, $86 \%$ belonged to Hindu religion and $80 \%$ were from rural areas. Literates and illiterates were almost equal in the study. Almost all of them belonged to lower class as per modified BG Prasad classification (Table 1).

Table 1: Socio demographic profile of the participants

\begin{tabular}{|l|c|c|}
\hline Characteristic & Categories & $\begin{array}{c}\text { Frequency/ } \\
\text { percentage }\end{array}$ \\
\hline \multirow{2}{*}{ Age in years } & $<60$ & 60 \\
\hline \multirow{2}{*}{ Gender } & $>60$ & 40 \\
\hline \multirow{2}{*}{ Religion } & Male & 31 \\
\cline { 2 - 3 } & Female & 69 \\
\hline \multirow{2}{*}{ Residence } & Hindu & 86 \\
\cline { 2 - 3 } & Other & 14 \\
\hline \multirow{2}{*}{ Education } & Rural & 80 \\
\cline { 2 - 3 } & Urban & 20 \\
\hline \multirow{2}{*}{ Socio economic class } & Illiterate & 46 \\
\cline { 2 - 3 } & Literate & 54 \\
\cline { 2 - 3 } & Lower middle & 6 \\
\cline { 2 - 3 } & lower & 48 \\
\hline
\end{tabular}

\section{Socio-cultural barriers}

Allopathic treatment was culturally acceptable in all the participants. All the participants had reported no fear of stigma towards their condition however one participant who was 32 years old was hesitant to reveal the diagnosis due to fear of stigma. Fasting was practiced by 34 participants amongst which 23 believed that taking medication would break their fast, hence they either skipped medication on the day of fasting or took medicines after they break the fast. $80 \%$ of the patients were dependent either economically or physically on their family members, amongst which only half of them got the required family support. The other $40 \%$ who had no support from family expected a monthly community based follow up at their home and free medications.

\section{Economic barriers}

63 patients are managing their monthly expenditure personally without depending financially on others. They spend around one fourth to half of their earnings for medication. $86 \%$ participants had no health insurance. They had faced both direct and indirect economic lose due to the condition. Direct loses included $60 \%$ of the participants being in debt and the indirect lose being $51 \%$ of them losing their daily wages. $83 \%$ of the participants felt that travel to hospital, stay and other expenses were adding on to their existing financial constraints.

\section{Educational barriers}

Participants were asked to list at least two precipitating factors and complications. They were also questioned about the knowledge regarding health facilities available for diabetic patients in and around their residence. Only $17 \%$ of the participants knew to check their blood sugar levels by themselves and all of them were literates. The proportion of people among literates who had good knowledge was higher as compared to illiterate but the difference was not statistically significant (Table 2).

Table 2: Knowledge on various components of diabetes among participants

\begin{tabular}{|l|c|c|}
\hline Knowledge component & Illiterates & Literates \\
\hline Precipitating factors & 16 & 43 \\
\hline Complications & 11 & 43 \\
\hline Availability of facilities & 43 & 51 \\
\hline Self-checking RBS & 0 & 17 \\
\hline
\end{tabular}

\section{Discussion}

Diabetes is a chronic condition and leads to complications due to non-compliance to treatment. Therefore identifying the barriers and addressing them becomes a necessity.

Allopathic treatment was culturally acceptable to everyone in the current study. In a study done in Kerala alternative medicine was opted over allopathy by $9 \%$ of the patients. All those who opted alternative therapy had no comorbities. The reasons quoted for the use of alternative medicine instead of allopathy were treatment with modern medicine being not effective and too toxic, alternative therapy was user friendly, free from adverse effects, available easily and in low cost. ${ }^{6}$ As the current study was hospital based, the patients admitted naturally tend to prefer allopathy due to complications faced. One of the female patient who was 32 years old, in the current study, has reported fear of stigma especially concerned with refusal in marriage proposals. In a study by Nancy et al., perceptions of stigma were significantly higher among respondents with type 1 diabetes than among those with type 2 diabetes and increased perception of stigma was associated with being female and having higher education levels. ${ }^{7}$ Another study revealed young adults with diabetes undergo all aspects of the social diabetes-related stigma as compared to older ones. $^{8}$ In the current study some of the patients practiced fasting without adequate knowledge about adjustment of medications. A study done on effects of fasting on diabetic patients reported that $10 \%$ experienced hypoglycemia, while $3.3 \%$ reported hyperglycemic episodes. There is a negative association between a visit to a physician by diabetic patients before they begin to fast regularly and the risk of developing hypoglycemia. Many diabetic patients who fast regularly are at high risk of adverse glycemic events. Most diabetics do not consult their physicians before fasting to adjust medications and lifestyle. ' Another study in Tamil Nadu concluded newer oral hypoglycemic agents and insulin with lower-risk profiles, permit stable patients to participate in fasts without major adverse consequences. With careful planning and education, patients may be able 
to participate in the number of feasts and fasts that dot the Indian calendar with the same vigor and zest. ${ }^{10}$ In the current study many participants reported dependency on family either economically or physically. But some of them were not getting the required support. A study proved that social support from family provides patients with practical help and can buffer the stresses of living with illness. ${ }^{11}$ In the current study diabetes caused both direct and indirect economic loses. A study done in Pune showed that $91.3 \%$ of indirect cost was due to loss of wages, in the current study though the percentage of expenditure is not estimated around half of the study participants faced loss of wages. ${ }^{12}$ A study done on economic burden of diabetes concluded excess health expenditures and lost productivity amongst individuals with diabetes, which varies in different settings and care. It suggested that innovative solutions are needed to cope with diabetes and its associated cost burdens in India. ${ }^{13}$ According to CURES study done in Chennai about $40.6 \%$ of the diabetics were aware of the complications ${ }^{14}$ but in our study $54 \%$ of people had good knowledge regarding the complications. Health literacy enhances the chances of a patient to be compliant to the therapy. Knowledge regarding the risk factors, complications would yield better self-care and avoid untoward outcomes.

\section{Conclusion}

Most of the study population belonged to lower strata. Social and economic dependency was the major barrier in the continuum of care. Fasting can be practiced after consultation with physician. Illiteracy and poor disease knowledge among the illiterates and literates is operating as a major barrier. Counselling sessions must be provided both in hospital and community based setting to enhance health literacy among diabetics.

\section{Source of Funding: None.}

\section{Conflict of Interest: None.}

\section{References}

1. Pawa M. Cultural influences and diabetes health care. Pract Diabetes In. 2003;20(6):200-1.

2. World Health Organization. Global Report on Diabetes. Geneva, Switzerland: WHO Press; 2016.

3. International Diabetes Federation - Facts \& figures [Internet]. Idf.org. 2019 [cited 18 November 2019]. Available from: https://www.idf.org/aboutdiabetes/what-is-diabetes/factsfigures.html

4. Worthington R, Gogne A. Cultural aspects of primary healthcare in India: A case- based analysis. Asia Pac Fam Medi. 2011;10(1):8.

5. Wangnoo S, Balaji V, Bantwal G, Das A, Dharmalingam M, Jain S et al. Barriers and solutions to diabetes management: An Indian perspective. Indian J Endocrinol Meta. 2013;17(4):594.

6. Vishnu N, Mini G, Thankappan K. Complementary and alternative medicine use by diabetes patients in Kerala, India. Glob Health, Epidemiol Genom. 2017;2.

7. Liu N, Brown A, Younge M, Guzman S, Close K, Wood R. Erratum: Stigma in People With Type 1 or Type 2 Diabetes. Clin Diabetes 2017;35:27-34. DOI: 10.2337/cd16-0020. Clin Diabetes. 2017;35(4):262.
8. Abdoli S, Abazari P, Mardanian L. Exploring diabetes type 1related stigma. Iran J Nurs Midwifery Res. 2013;18(1):65-70.

9. Noon M, Khawaja H, Ishtiaq O, Khawaja Q, Minhas S, Niazi A et al. Fasting with diabetes: a prospective observational study. BMJ Glob Health. 2016;1(2):e000009.

10. Mahadevan S, Kannan S, Seshadri K, Sadacharan D, Velayutham K. Fasting practices in Tamil Nadu and their importance for patients with diabetes. Indian J Endocrinol Metab. 2016;20(6):858.

11. Miller T, DiMatteo R. Importance of family/social support and impact on adherence to diabetic therapy. Diabetes Metab Syndr Obes: $2013 ; 421$.

12. Gogate $\mathrm{P}$, Chandra $\mathrm{P}$, Gogate B, Thite N, Mutha A, Walimbe A. Economic Burden of Diabetes in Urban Indians. The Open Ophthalmol J. 2015;8(1):91-4.

13. Sharma K, Ranjani H, Zabetian A, Datta M, Deepa M, Moses $\mathrm{C}$ et al. Excess cost burden of diabetes in Southern India: a clinic-based, comparative cost-of-illness study. Glob Health Epidemiol Genom. 2016;1.

14. Mohan D, Raj D, Shanthirani CS, Datta M, Unwin NC, Kapur A et al. Awareness and knowledge of diabetes in Chennai--the Chennai Urban Rural Epidemiology Study [CURES-9]. $J$ Assoc Physicians India. 2005; 53:283-7.

How to cite this article: Manjunatha SN, Jayashree S, Perceived challenges in diabetic care: a cross sectional study among inpatients of a tertiary care hospital, Indian J Forensic Community Med 2019;6(4):208-10. 